Aims Rheumatic heart disease (RHD) is a potentially debilitating and fatal condition with a high prevalence in low resource countries. The Gambia has many individuals with severe, symptomatic RHD but limited cardiology and no cardiothoracic surgical services. Charitable organisations fund surgery overseas for a minority. We aimed to review post-operative outcomes in this group.

Methods Paediatric RHD patients with documented pre- and post-operative management at our institution were included. Retrospective data from clinical records was collected on age, sex, HIV, HBV and sickle cell status, operation type, pre- and post-operative Ross scores and medication burden (table 1). For warfarinised patients, 'percentage days within therapeutic range' was calculated using the Rosendaal method.

Results 17 patients matched our inclusion criteria; 11 male, 6 female. Median age at surgery was 16 years. There was one death (cause: infective endocarditis). In total, 19 operations were performed involving 31 valve procedures; 7 metallic and 5 bioprosthetic valve replacements and 19 repairs.

\begin{tabular}{llll} 
Abstract G270(P) Table 1 & & \\
\hline & $\begin{array}{l}\text { Pre-operative median } \\
\text { (IQR) }\end{array}$ & $\begin{array}{l}\text { Post-operative median } \\
\text { (IQR) }\end{array}$ & $\begin{array}{l}\text { P } \\
\text { value }\end{array}$ \\
\hline Ross Score & $2(2-3)$ & $1(1-1)$ & 0.013 \\
$\begin{array}{l}\text { Number of Pills per day } \\
\text { Daily Furosemide dose }\end{array}$ & $3(2-5)$ & $1(0-2)$ & 0.004 \\
(mg) & $20(10-40)$ & $0(0-20)$ & 0.242 \\
$\begin{array}{l}\text { Daily Spironolactone dose } \\
\text { (mg) }\end{array}$ & $25(0-25)$ & $0(0-0)$ & 0.075 \\
Daily captopril dose (mg) & $37.5(5-50)$ & $0(0-50)$ & 0.279 \\
\hline
\end{tabular}

For the six warfarinised patients, median percentage days within target therapeutic range (TTR) was $40.2 \%$ and percentage of tests within TTR was 34.1\%.

Conclusion In this small cohort of Gambian children and adolescents, cardiac surgery had a positive impact with significant effects on exercise tolerance and pill burden. Normal exercise tolerance following fifteen (78.9\%) operations had positive social, economic and medical implications. However, there is a long-term risk of haemorrhage and thromboembolism in warfarinised patients given the low proportion of time in therapeutic range. Our evaluation highlights the challenges of working with adolescents around medication adherence, lack of alternatives to warfarin for young women of child bearing age and the need for high quality pre- and post-operative assessment and follow-up in low resource environments.

\section{G271(P) IMPROVING PRACTICE OF PAEDIATRIC ADMISSION VITAL SIGN MEASUREMENT: AN AUDIT OF VITAL SIGN DOCUMENTATION FOR PATIENTS ADMITTED TO A LARGE PAEDIATRIC MEDICAL WARD BEFORE AND AFTER DELIVERY OF A PAEDIATRIC ASSESSMENT EDUCATION PROGRAMME}

\footnotetext{
1,2A Tailby, ${ }^{1,2} \mathrm{M}$ Macken, ${ }^{1,2} \mathrm{~W}$ Collett. 'Malawi Partnership, Birmingham Children and Women's Hospital Foundation Trust, Birmingham, UK; ${ }^{2}$ Paediatric Department, Queen Elizabeth Central Hospital, Blantyre, Malawi
}

10.1136/archdischild-2018-rcpch.264
Aims This audit aimed to quantify the impact that delivering a Paediatric Assessment Skills and Leadership (PAS) course would have on the measurement and documentation of patients' vital signs on admission to an inpatient department.

Methods Data was collected from the paediatric medical ward over seven days. Notes for each admission in the past 24 hours were examined for the recording of vital sign parameters. The standard for data inclusion was from the local Critical Care Pathway which defines eight base line observations to be recorded on each admission to the department. Over the following two weeks a PAS course was delivered to 34 nurses. The course taught clinical examination skills and a Train the Trainer programme to empower the local team to deliver the course in the future. One month after the course a re-audit was taken on the same ward. To avoid bias neither audit timings were disclosed to the ward staff.

Results In the initial audit, notes for $97 \%$ of the 234 admissions $(n=227)$ were examined. Of these only $1.76 \%(n=4)$ had a full set of documented vital signs. Blood pressure was the least recorded $(2.4 \%)$, respiratory rate was recorded in less than half of all admissions (45.3\%) and temperature was the highest documented vital sign (96.5\%). After delivery of the PAS course the re-audit captured $100 \%(n=28)$ of admissions in a 24 hours period and found that a full set of vital signs were documented in 32\% of all admissions (an increase of $30.24 \%$ ), blood pressure measurement increased by $34 \%$ and respiratory rate was documented in $75 \%$ of all admissions.

Conclusion The PAS course had a positive impact on the frequency of documentation of admission vital signs. Further improvement is required to ensure all children receive a comprehensive documentation of vital signs. This may be achieved through on-going education. Future audits should be carried out to assess the development of the service and act as an incentive to maintain high standards. For sustainability the responsibility of the audit, feedback and education process should be given to the leaders within the local team.

\section{G272(P) ABSTRACT WITHDRAWN}

\section{G273(P) SUCCESSES AND CHALLENGES OF INTRODUCING CLINICAL JOB AIDES IN DISTRICT HOSPITALS IN MYANMAR}

1,2J Halbert, ${ }^{2} \mathrm{M}$ Malley, ${ }^{2} \mathrm{M}$ Monaghan, ${ }^{2} \mathrm{E}$ Roche, ${ }^{2} \mathrm{P}$ Babu, ${ }^{2} \mathrm{~S} \mathrm{Oo},{ }^{2} \mathrm{~J}$ Bowyer, ${ }^{2} \mathrm{M}$ Wooton, ${ }^{2}$ KT Sin. ${ }^{1}$ Paediatrics, University College London Hospital, London, UK; ${ }^{2}$ Global Child Health, Royal College of Paediatrics and Child Health, London, UK

10.1136/archdischild-2018-rcpch.265

Aims Clinical job aides directly benefit clinical care and assist project evaluation. We describe early experience of their introduction as part of an Emergency Paediatric Care Programme (EPCP).

Methods An EPCP working group developed pilot job aides. These included admission, observation and drug charts that were piloted in 11 hospitals. Between May and October 2016 the job aides were reviewed and a survey of local healthcare workers undertaken.

Results In the majority of hospitals job aides were limited or inadequately completed. Vital sign charts typically only 
recorded the temperature. The prescription and administration of drugs were recorded in separate places, and admission information recorded on a brief proforma.

Although the job aides received positive endorsement during the EPCP courses there has been some resistance to their use in the wards. A survey of patient notes at one site demonstrated that no staff were using the admission forms or the drug charts adequately. Vital signs charts were more acceptable and $100 \%$ were satisfactory.

Healthcare workers considered the job aides useful, but cited poor staffing and lack of time as the reason for limited acceptance. Suggestions included:

- Senior endorsement

- Training for all staff on how to use the job aides

- Explanation to nurses how vital signs charts can be used to monitor patient progress and signal when to get help

Conclusion Job aides can focus the attention of healthcare workers on key signs and symptoms as taught in EPCP. Ongoing training, familiarisation, mentorship and support from senior colleagues will be key for their successful introduction.

\section{G274(P) STABILISATION, REFERRAL \& TRANSFER OF ACUTELY UNWELL CHILDREN IN MYANMAR}

${ }^{1,2} \mathrm{~J}$ Halbert, ${ }^{2} \mathrm{M}$ Malley, ${ }^{2} \mathrm{M}$ Monaghan, ${ }^{2} \mathrm{E}$ Roche, ${ }^{2} \mathrm{P}$ Babu, ${ }^{2} \mathrm{~S} \mathrm{Oo},{ }^{2} \mathrm{~J}$ Bowyer, ${ }^{2} \mathrm{M}$ Wooton. ${ }^{1}$ Paediatrics, University College London, London, UK; ${ }^{2}$ Global Child Health, RCPCH, London, UK

\subsection{6/archdischild-2018-rcpch.266}

Aims An Emergency Paediatric Care Programme in Myanmar provides training in the stabilisation and safe transfer of sick children. The transfer of seriously sick children may be necessary to ensure the appropriate level of care, but is fraught with difficulties. A review of current practice and local challenges was undertaken.

Methods In September 2015 a retrospective audit of children admitted with dengue to a Regional Centre was performed to identify reasons for referral. Local healthcare professionals were invited to complete a survey exploring barriers to referral pathways.

Results $34 \%$ of patients with dengue $(n=269)$ were referred. Only $44 \%$ of referrals had a referral letter and $35 \%$ did not require tertiary care.

Challenges identified included:

- Late presentation due to families' preference for traditional medicine, concerns about the quality of public healthcare, medical and transport costs

- Lack of referral guidelines, human resources and training

- Transfer of patients who had not first been stabilised

- Reluctance to acknowledge the potential benefits of transfers

- Deficiency in ambulances such that patients were transferred by public transport

Solutions identified and implemented included:

- Development of referral guidelines and pathways

- Training in stabilisation and transfer

- Development of transfer checklists for emergency equipment and medications

- Identification of voluntary sector transport vehicles
- Identification of outpatient tertiary clinic appointments to reduce unnecessary acute referrals

Conclusions There is a deficiency in referral pathways between primary, secondary and tertiary healthcare facilities in Myanmar. Development of referral networks and the training of healthcare workers in the stabilisation and transfer of sick children is important to reduce morbidity and mortality.

\section{G275(P) A STRATEGY TO SUPPORT CHANGES IN CLINICAL CARE FOLLOWING TRAINING COURSES AT DISTRICT HOSPITALS IN MYANMAR: IMPLEMENTATION PLANS}

1,2J Halbert, ${ }^{2} \mathrm{M}$ Malley, ${ }^{2} \mathrm{M}$ Monaghan, ${ }^{2} \mathrm{E}$ Roche, ${ }^{2} \mathrm{P}$ Babu, ${ }^{2} \mathrm{~S} \mathrm{O} 0,{ }^{2} \mathrm{~J}$ Bowyer, ${ }^{2} \mathrm{M}$ Wooton, ${ }^{2}$ KT Sin. ${ }^{1}$ Paediatrics, University College London, London, UK; ${ }^{2}$ Global Child Health, RCPCH, London, UK

\subsection{6/archdischild-2018-rcpch.267}

Aims An Emergency Paediatric Care Programme (EPCP) runs training courses in district hospitals. We describe a strategy to support local leadership and develop teams responsible for devising and fulfilling individual implementation plans.

Methods During EPCP courses, participants review the hospital facilities and make recommendations for improvement. A local EPCP Champion (senior clinician) supported by an EPCP Team is responsible for identifying local priorities and developing an Implementation Plan to achieve these. They are asked to consider: layout, infrastructure, staffing, patient safety and infection control, drugs and equipment, staff numbers and training, patient records, clinical guidelines, and morbidity and mortality meetings. Additional support is provided through Global Link Volunteer (GLV) placements in the hospitals.

A bi-annual EPCP Champion and Team Conference provides an opportunity to share ideas and experiences, problem solve and discuss quality improvement projects.

Results GLVs, EPCP Champions and Teams representing 7 district hospitals reported on their Implementation Plans at the first EPCP Champion and Team Conference in July 2016. A wide range of topics were described including weekly simulation training, new guideline wall charts, well-equipped resuscitation areas, new admission and observation charts, improved infection control through the repair of broken sinks and provision of hand-gel and multi-disciplinary mortality meetings.

Feedback was extremely positive with the teams feeling motivated, empowered and rewarded for their efforts.

Conclusions Successful training depends on its impact in practice; the EPCP Champions and Team Conference demonstrated that the combined efforts of local EPCP Champions and Teams and GLVs in developing the Implementation Plans had led to valuable quality improvements.

\section{G276(P) NEONATAL MORTALITY IN WESTERN UGANDA}

${ }^{1} \mathrm{C}$ Harris, ${ }^{2} \mathrm{H}$ Patel, ${ }^{2} \mathrm{O}$ Corn, ${ }^{2} \mathrm{U}$ Harris, ${ }^{2} \mathrm{M}$ Munyagwa, ${ }^{1} \mathrm{~A}$ Greenough. ${ }^{1}$ Women and Children's Health, School of Life Course Sciences, Faculty of Life Sciences and Medicine, King's College London, London, UK; ${ }^{2}$ Kagando Hospital, Uganda

\subsection{6/archdischild-2018-rcpch.268}

Aims Neonatal mortality in Uganda has not improved in the last 14 years and remains between 24 and 27 deaths per 1000 live born infants, which compares poorly to the UK neonatal mortality (2.4 per 1000 live born infants). Our aim 